\title{
Comparison of Exercise Open Kinetic Chain and Closed Kinetic Chain in The Rehabilitation of Patellofemoral Dysfunction: an Updated Revision
}

\author{
Thatiana Lacerda Nobre
}

Physiotherapist, graduated from Catholic University of Santos / BRAZIL. Post graduate degree: Physiological and Methodological Basis of Athletic Training and Locomotion system in Sports - Federal University of São Paulo / BRAZIL

thatianalacerda@yahoo.com.br

\begin{abstract}
Introduction: The patellofemoral syndrome (PDS) is a disease characterized by pain in the patellofemoral 1 and physical changes resulting from this joint biomechanics. The rate of incidence is high and its etiology remains unknown. Objective: This literature review aims to compare the effectiveness of open kinetic chain exercises with closed kinetic chain into the functional recovery of patients with the patella-femoral syndrome. Methods: We conducted a systematic review in the database LILACS, MEDLINE and PUB MED SCIELO as search term words patellofemoral syndrome, Open Kinetic Chain and Closed Kinetic Chain. Discussion: The imbalance of forces of patella dynamic stabilizers, Vastus medialis oblique (VMO) and vastus lateralis (VL), is still considered the primary factor for the onset of symptoms. Since this change causes an increase in reaction force and patellofemoral compression. In order to recover the balance of forces of the muscles acting as stabilizers of the knee and as a means of restoring stability to the joint, exercises in open kinetic chain (OKC) and closed kinetic chain (CKC) has been used in rehabilitation programs patellofemoral disorders. Conclusion: The benefits for implementing these protocols are not well documented; scientific evidences are insufficient to prove the real effectiveness of these exercises in improving the performance of quadriceps muscle or assist in muscle balance of patellofemoral stabilizers.
\end{abstract}

Keywords Rehabilitation Syndrome, Open Kinetic Chain, Closed Kinetic Chain, Patellofemoral Dysfunction, Patellofemoral Force

\section{Introduction}

The patellofemoral dysfunction is one of the muscular-sk eletal disorders frequently knee $(1,2,3,18)$. Its incidence rate is about $25 \%$ of disorders in the population $(19,20)$. Symptoms consist of diffuse pain in the anterior knee, most are usually along the medial aspect of the patella. However, pain retro patella and the lateral face can also be diagnosed (4). These symptoms are caused by structural orbiomechani cal changes of the joint, which becomes exacerbated by activities such as going up and down stairs, sit for a prolonged period, squatting or kneeling $(2,5,21)$, resulting in increased compressive forces in the joint patellofemoral (4, 6). Other signs are also present as the patellar crepitus, swelling, and joint blockage (6).

This disease affects athletes and non-athletes andreprese nts a common problem in the knee of adolescents and young adults physically active $(5,6,7)$. The factors that contribute

* Corresponding author:

thatianalacerda@yahoo.com.br (Thatiana Lacerda Nobre)

Published online at http://journal.sapub.org/cmd

Copyright $(2012$ Scientific \& Academic Publishing. All Rights Reserved to the development of this pathology is still not clearly defined. $(2,3,6,20,22)$. The mechanism may be multifaceted $(22,23)$. However, it may be related to abnormal biomecha nics, highlighting, among these, the static and dynamic unbalance $(1,21)$.

Changes in static, some authors suggest some abnormaliti es such as patellar misalignment, increased Q angle, patella high or low, excessive subtalar pronation, lateral rotation of the tibia, femoral anteversion, knee valgus or varus and shortening of the lateral retinaculum, muscle hamstrings and iliotibial tract $(1,6)$. Thus, inappropriate behavior of the patella can cause pain in the anterior knee (5).

However, Connolly and colleagues (20), the explanation for the origin of patellofemoral pain is related to three main factors, such as patellar misalignment, muscle unbalance and overuse. These factors lead to the region to an increase in stress on the soft tissues and consequently pain.

Towards Frederickson \& Yoon (19), suggest that a combination of factors, such as abnormal lower limb biomechanics, soft tissue tension, muscle weakness and excessive exercise may result in increased stress on the cartilage and subchondral bone. Therefore promote a patellar misalignment and the development of pathology. 
The patellar surface of the femur is the anterior articular portion which articulates with the patella and is divided into two facets: the lateral and medial. The lateral facet is, in general, more and more previously designed, in most people, the medial facet (8). It is noted a hollow in its anatomy in two planes, promoting intimate contact with the lateral femoral condyle, while most of the range of motion (9). This helps maintain the facet of the patella centered on the patellar surface of femur during the normal function of the knee. When there is instability of the patella, there is a variation of the patellar surface of femur (8). Already the medial facet is small quantity convex, and only a small portion of its surface comes into contact with the medial femoral condyle (9).

The instability can be observed with increased Q angle, which promotes an increase in lateral patella (21), promoting an increased stress on the patellofemoral joint $(18,23)$.

Articular cartilage covers the surface of the femoral patellar is thinner than the patella, and also thinner medial facet on the side (8). The patellar cartilage is more permeable and resilient when compared with other cartilage body. Thus, the load, its contact area increases, thereby decreasing the pressure (10).

The patella is the central point to which converge the elements retinacular, ligaments, muscles, tendons and synovial capsule (8). Because of the inconsistency and the ability to move in relation to the femur, the contact point on the patella changes with flexion or knee extension (10).

During the full extent and with the quadriceps contracted, the patella articulates with the suprapatellar fat pad, and when in full flexion, with part of the medial and lateral femoral condyle that articulates with the tibial plateau at full extension (8).

The dynamic traction of the patella is affected by a number of forces that tend it moves both laterally and medially. These forces come into play when the nervous system activates the control of the muscles acting on the patella (11).

The main structure responsible for activating the forces exerted on the patella is the quadriceps muscle, whose function is to control the position of the patella from the trochlea by the oblique fibers of their medial and lateral portions, and the vastus medialis (VM ) and vastus lateralis (VL) (11).

The VM muscle is divided into two portions, a proximal called the vastus medialis longus (VML) and distal sites, the vastus medialis oblique (VMO). These parts show anatomical, functional, histochemical and also in the pattern of innervation (1). The lateral vastus medialis (VML) is inserted at an angle of 15 degrees to the longitudinal axis of the femur, exerting little or no traction for proper positioning of the patella and the vastus medialis oblique (VMO), which originates mainly from tendon of adductor magnus muscle and is inserted at an angle of 50-55 degrees in the longitudinal axis of the femur (11) and is considered the primary dynamic medial stabilizer of the patellofemoral joint $(11,22)$.

Similarly, the VL muscle is also divided into two portions: the proximal fibers that originate in the femur and insert on the medial third of the tendon of the quadriceps, constituting the vastus lateralis longus (VLL), and fiber posterolateral that originate in the iliotibial tract, being more oblique in its direction, and fall at the base and the lateral edge of patella, representing the vastus lateralis obliquus (VLO) (11).

Considering the origins and insertions of the $\mathrm{VM}$ and $\mathrm{VL}$ muscles, especially the oblique portions thereof, may be considered that the pull of the VMO and VLO is important because muscle is the factor that determines the position of the patella. These muscles also exhibit behavior synchronous with an antagonistic role in patellar stabilization $(1,8)$. Under normal conditions, the VMO is able to counteract the action of the VL lateralization, to ensure the stability of the patella (22).

The force exerted by the VLO is related to the lateral retinaculum and iliotibial tract, and may be able to alter the normal balance of the patella, thus creating excessive tension in these structures and may prompt a picture of pain and patellar misalignment (1). The delayed onset and decreased magnitude of activation of the VMO relative to VL leads to an abnormal lateral movement and increased patellofemoral contact pressure is resulting in the articular cartilage pathology. The reduction of power production capacity of the VMO or change in motor control of VMO and VL has been proposed as possible causes of imbalance $(4,22)$, between the dynamic stabilizers of the knee (22).

The imbalance of the dynamic stabilizers is related to the forces between the Vastus medialis oblique (VMO) and vastus lateralis $(\mathrm{VL})(3,5)$, the main dynamic stabilizers of the patella, this imbalance is considered the primary factor for the onset of symptoms, which, however, change the kinematics and patellar contributes to increase the forces of reaction and patellofemoral compression $(3,22)$.

Misalignment of the extensor mechanism is a very common feature of this disease by promoting atrophy and decreased strength of the vastus medialis and imbalance between the lateral and medial components of the quadriceps $(1,22)$.

Frederickson \& Yoon (19) report that, the stresses exerted on the quadriceps muscle, promote high stress in the patellofemoral region. Similarly, the deficit on the flexibility of the quadriceps and hamstrings, show an association with patellofemoral pain.

The kinetic chain is a term used to describe the sequential activation of the leg segments of the limb, allowing it to generate force, stabilization of the leg, and transfer force to the distal end of the chain (12).

An exercise in open kinetic chain can be considered an activity in which the distal part of the end is not fixed, but free space $(4,13)$, providing a motion segment in isolation (6). Furthermore, it is considered that usually this type of exercise no weight bearing (12).

The closed kinetic chain exercises involving multi-joint movements performed with fixed distal extremity $(4,13)$, often associated with weight bearing $(4,12)$.

These exercises generate muscle co-contraction of agonists and antagonists, in order to provide greater articular 
$(3,13)$ producing still lower shear load of the tibia, the compression force increasing and decreasing thetibia-femor al compressive forces near patellofemoral the extension (14). For Fagan \& Delahunt (4), proprioception is also an influential factor in the choice of these exercises, since it is believed that the feedback is more efficient due to the compressive forces of the body and the foot contact with the ground, and reproduce functional movements commonly performed activities of daily living $(3,4,7)$.

The literature data on the comparison of these two chains are still present inconclusively. Moreover, the benefits to the application of these protocols are not well documented. Thus, the objective of this study is to review the literature to compare the effectiveness of open kinetic chain exercises with closed kinetic chain for functional recovery of patients with dysfunction of the patellofemoral.

\section{Methodology}

For this study, respondents were magazine articles that are accessed through the portal database journals Lilacs, Medline, Scielo and Pub med, since 1998 until 2011. The strategy seeks to correlate the following words - key: patellofemoral rehabilitation, open kinetic chain, closed kinetic chain, patellofemoral syndrome, patellofemoral forces, patellofemoral biomechanics. The languages were analyzed in Portuguese and English. Inclusion criteria for the selection of the papers surveyed, we took into account the relationship with the pathology and the exercises, and the date of publication of these studies.

\section{Discussion}

This study consists of a literature review concerning this subject. Thus, the found results in the articles cited, are presented in this session.

During the open kinetic chain exercise, the quadriceps muscle works in isolation, increasing the patellofemoral compressive forces (3). In this type of exercise, the center of gravity is forward of the knees, so that the strength in flexion is greater than 90 degrees to $0^{\circ}$ extension (10). Up to $30^{\circ}$, the angle between the forces is too small to generate high compressive stress between the patella and the condyles and the contact area decreases from $90^{\circ}$ to $0^{\circ}$. With a larger force and a smaller contact area, the pressure is maximum around $35^{\circ}$ to $45^{\circ}$, decreasing as a result because the angle is very small. So, exercise in open kinetic chain can be performed from $0^{\circ}$ to $15^{\circ}$ and $50^{\circ}$ to $90^{\circ}$ in the individual with DFP, not realizing the financial year $35^{\circ}$ to $45^{\circ}$, where the contact pressure is very high, since the magnitude of the contact patellofemoral grows from $0^{\circ}$ to $60^{\circ}$ and does not change much from $60^{\circ}$ to $90^{\circ}(10)$. According to Fehr et al (3) must be avoided the last degrees of knee extension, since this angle is less articular contact, however, the compressive forces are distributed over a small area, increasing the patellofemoral stress. According to Grossi et al (1), the last degrees of knee extension exercise in open kinetic chain, provide lower articular contact and therefore less instability. There is also higher patellofemoral stress, since the contact angle is less and therefore the compressive forces although smaller, are distributed over a smaller contact area, increasing the stress.

The concept that the VMO is most active during the last degrees of extension is widely accepted by Grossi, Peter and Berzin (1) and Escamilla and colleagues (15). Because this muscle is difficult to isolate, an exercise - conducted in open kinetic chain - proposed by O 'Sullivan \& Popelas (2) in their study showed that in the last degrees of knee extension with medial rotation of the tibia obtained - better activation VMO.

In the closed kinetic chain exercise, the center of gravity is located behind the knee, so the force is increased from $0^{\circ}$ to $90^{\circ}$. This increase in strength is accompanied with an increased contact area to $60^{\circ}$ and, thereafter, the contact area does not increase in proportion to the force, causing a pressure increase in the patella (10). The knee flexion to $90^{\circ}$ presents a greater patellofemoral reaction force, being larger, also the patellofemoral articular contact and stability. Thus, the patellofemoral stress decreases with increasing angle of knee flexion (1).

According to Grossi et al (5), the closed kinetic chain exercises in the first $60^{\circ}$ of knee flexion is better tolerated by individuals with DFP. For Souza et al (13) in the range of $0^{\circ}$ to $50^{\circ}$ of knee flexion occur under anterior shear forces on the tibiofemoral joint. Fehr et al (3) say should be avoided angles above $45^{\circ}$ of knee flexion, since despite more stable joint with increased flexion there are also increased compressive forces and increased patellofemoral stress. Peccin \& Chamlian (16) indicate that, from $0^{\circ}$ to $30^{\circ}$ of hip flexion, the patellofemoral reaction forces decrease, and this joint stress compared with exercise in open kinetic chain. The closed kinetic chain activities, however, must be an angle between $0^{\circ}$ and $30^{\circ}$, for $60^{\circ}$ to $90^{\circ}$ there is an increase of stress on this joint. Have Cabral et al (6) report that the exercises performed in the early degrees of flexion also cause less lateral patellar traction.

In this type of exercise there is greater amplitude of the signal of the VMO compared with the open kinetic chain exercises. However, the signal VMO is lower than that of VL, both in open and closed kinetic chain (6). To selectively strengthen the VMO muscle, which seeks not only the range of motion that shows more activity, but also offers greater stability, higher compressive strength and greater activation in relation to the lateral components. In 90 degree angle, the compressive forces are maximized and the shear force is minimized, facilitating not only the activity of the VMO muscle, but increased the articular contact facilitating articular nutrition (1).

In the closed kinetic chain exercise occurs co-contraction hamstring. In small angles of flexion such co-contraction decreases the tibial anterior and quadriceps caused by inward rotation, however, at angles above $60^{\circ}$ co-contraction this 
causes the tibia to rotate and move further outwardly. This posterior displacement and external rotation increased the pressure on the patella, as well as the contact force is greater than $50^{\circ}$ co-contraction hamstrings and increase the pressure from 60 (10).

Among the $\mathrm{CKC}$ exercises, the squat is considered safe and effective, due to the stabilizing effect of co-contraction quadriceps and hamstrings (5). This exercise should be performed to near 50 degrees, not to generate as much force and pressure in the patellofemoral joint (10).

During squatting, the line of gravity moves after the knee axis, the flexor increasing the torque. The hamstrings stabilize the knee by promoting a draw later in the tibia to counteract the force imposed by the previous quadriceps.

The degree of activation in the hamstrings work in the pelvis depends on the knee and hip angles and muscle lengths of the individual. Thus, the hamstring tension may be increased with a slight flexion of the trunk, which also moves the center of gravity above, reducing the torque and knee flexion, thereby reducing the strength of tibia translation and compression of the patellofemoral joint (13).

Grossi et al (5) observed that with increasing knee flexion using the technique of the squat that provides higher electrical activation of the VMO and VL. This occurs because of the rectus femoris to be more active in this chain and, consequently, the VMO increases its electrical activity to keep the patella in its proper alignment. In general, the quadriceps muscle electrical activity is more that increases as the angle of knee flexion. In a study proposed by O'Sullivan \& Popelas (2) it was observed that the squat with external rotation of hip and knee flexion to $60^{\circ}$ produces a greater degree of activation of the VMO muscle.

ZWERVER et al (17) in their study reported that squat platform held in a decline in an eccentric way proved to be more effective for strengthening the extension of the knee region compared with the normal and squat on level ground. The authors believe that is the fact that in both standing and on the decline occurs when the reduction of the strength of the hip and ankle during exercise. Thus, the knee extensors and the patellar tendon is loaded to the maximum when applied to a plate 15 to $30^{\circ}$. The knee flexion should be avoided $60^{\circ}$ because this angle patellofemoral contact is already about nine times the force of the body. Therefore, to avoid patellofemoral syndrome, is recommended to avoid knee flexion during the squat exercise above $60^{\circ}$.

The activation of the rectus femoris muscle was found to be maximized with increasing knee flexion angle of $90^{\circ}$. Looking at the closed kinetic chain exercises, the greater activity of the rectus femoris is produced between $83^{\circ}$ and $95^{\circ}$ of knee flexion. This angle increases the compressive forces tibiofemoral and patellofemoral joints (14).

\section{Conclusions}

After this survey we can conclude that among the treatment protocols, exercises in open and closed kinetic chain shown to reduce pain and increase muscle strength of patients with this pathology. The therapeutic approach combined with these two chains is recommended. But the exercises in closed kinetic chains have been considered more functional for the rehabilitation of individuals with DFP. However, they lack scientific evidence to prove the real effectiveness of these exercises to improve the performance of the quadriceps muscle and help balance the dynamic stabilizers of the patella. Thus, they become more necessary biomechanical, electromyographic and anatomic allowing a comparison between them in order to base the application of exercise in the treatment of patients with this pathology.

\section{REFERENCES}

[1] GROSSI DB, PEDRO VM, BERZIN F. Análise funcional dos estabilizadores patelares. Acta Ortopédica Brasileira. 2004;12 [2]: $99-104$.

[2] O' SULLIVAN SP, POPELAS CA. Activation of vastus medialis obliquus among individuals with patellofemoral pain syndrome. Journal of strenghth and conditioning research. 2005; 19 [2]: $302-304$.

[3] FEHR GL, JUNIOR, AC, CACHO EWA, MIRANDA JB. Efetividade dos exercícios em cadeia cinética aberta e cadeia cinética fechada no tratamento da síndrome da dor femoropatelar. Revista Brasileira de Medicina do Esporte. $2006 ; 12$ [2]:66 - 70.

[4] FAGAN V, DELAHUNT E. Patellofemoral pain syndrome: a review on the associated neuromuscular deficits and current treatment options. British Journal Sports Medicine. 2008; 42: 789-795.

[5] GROSSI DB, FELICIO LR, SIMÕES R, COQUEIRO KRR, PEDRO VM. Avaliação eletromiográfica dos músculos estabilizadores da patela durante exercício isométrico de agachamento em indivíduos com Síndrome da dor femoropatelar. Revista Brasileira de Medicina do Esporte. 2005; 11 [3]: $159-163$.

[6] CABRAL CMN, MELIM AMO, SACCO ICN, MARQUES AP. Fisioterapia em pacientes com síndrome fêmoro - patelar: Comparação de exercícios em cadeia cinética aberta e fechada. Acta Ortopédica Brasileira. 2008; 16[3]: 180 - 185.

[7] WITVROUW, E, DANNEELS L, TIGGELEN DV, WILLEMS TM, CAMBIER D. Open versus closed kinetic chain exercises in patella-femoral pain. A 5- year prospective randomized study. The American Journal of Sports Medicine. 2004; 32 [5]:1122 - 1130.

[8] COHEN M, ABDALlA RJ, FILHO MF, SILVA PRG. Síndrome femoropatelar. In: COHEN, M.; ABDALA, R. Lesões no esporte Diagnóstico - Prevenção - Tratamento. Rio de Janeiro: Revinter, 2002.

[9] TUREK, S. L. Ortopedia - princípios e sua aplicação. São Paulo : Manole, 1991.

[10] HAUPENTBAL A, SANTOS DP dos. Força e contato patelofemoral como fundamentos biomecânicos para reabilitação da síndrome patelofemoral. Fisioterapia em 
Movimento. 2006; 19 [4]: 11-16.

[11] CABRAL CMN, MONTEIRO PV. Recuperação funcional de indivíduos com disfunção fêmoro - patelar por meio de exercícios em cadeia cinética fechada: Revisão de literatura. Revista Brasileira de Fisioterapia. 2003; 7 [1]:1-8.

[12] DAVIES, G. J.; HEIDERSChEIT, B. C.; CLARK. M. Reabilitação em cadeia cinética aberta e fechada. In: ELLENBECKER TS. Reabilitação dos ligamentos do joelho. Manole, 2006.

[13] SOUSA CO de.; eta al. Atividade eletromiográfica no agachamento nas posições de $40^{\circ}, 60^{\circ}$ e $90^{\circ}$ de flexão do joelho. Revista Brasileira de Medicina do Esporte. 2007;13 [5]: $310-316$.

[14] FLEMING BC, OKSENDAHL H, BEYNNON BD. Open- or Closed-Kinetic Chain Exercises After Anterior Cruciate Ligament Reconstruction. Exercise and Sport Sciences Reviews. 2005; 33 [3]:134 - 140.

[15] ESCAMILLA RF, FLEISIG GS, ZHENG N, BARRENTINE SW, WILK KE, ANDREWS JR. Biomechanics of the knee during closed kinetic chain and open kinetic chain exercises. Medicine and Science in Sports and Exercise. 1998; 30 [4]: 556 - 569 .

[16] PECCIN MS, CHAMLIAN TR. Princípio da reabilitação femoropatelar. In: COHEN, M, ABDALA R. Lesões nos esportes - Diagnóstico - Prevenção -Tratamento. Rio de
Janeiro: Revinter, 2002.

[17] ZWERVER J, BREDEWEG SW, HOF AL. Biomechanical analysis of the single-leg decline squat. British Journal Sports Medicine. 2007; 41: $264-268$.

[18] MARTÍN MC, SANTIAGO, FR, CALVO RP, ÁLVAREZ LG. Patellofemoral morphometry in patients with idiopathic patellofemoral pain syndrome. European Journal of Radiology. 2010; 75 [1]: $64-67$.

[19] FREDERICSON M, YOON K. Physical examination and patellofemoral pain syndrome. Am J Phys Med Rehabil. 2006; 85 [3]: $234-243$.

[20] CONNOLLY KD, RONSKY JL, WESTOVER LM, KUPPER JC, FRAYNE R. Differences in patellofemoral contact mechanics associated with patellofemoral pain syndrome. Journal of Biomechanics. 2009; 42: 2802 - 2807.

[21] SHEEHAN FT, DERASARI A, FINE KM, BRINDLE TJ, ALTER KE. Q-angle and J-sign. Clin. Orthop. Relat. Res. 2010; 468: $266-275$.

[22] PATTYN E, et al. Vastus medialis obliquus atrophy: Does it exist in patellofemoral pain syndrome. The American Journal of Sports Medicine. 2011; 39 [7]: 1450 - 1455.

[23] LONG-ROSSI F, SALSICH GB. Pain and hip lateral rotator muscle strength contribute to functional status in females with patella-femoral pain. Physiotherapy Res Int. 2010; 15 [1]: 57 -64 . 